\title{
Utilisation des plutéus en écologie expérimentale
}

\author{
Paul Bougis \\ Station Zoologique, Villefranche-sur-Mer, France
}

\begin{abstract}
The use of pluteus-larvae in experimental ecology. The pluteus of sea urchins (Paracentrotus lividus and Arbacia lixula) are planktonic organisms easily reared in the laboratory for experimental purposes. After a short summary of methods, the stages of development are outlined. These include an endotrophic phase, an exotrophic phase and a period of regression. Requirements for experimental use are described, and some applications are then shown. The latter include estimates of toxicity (copper, bactericidal solutions), and measures of food value (cultures of unicellular algae). Some prospects of using these pluteus in experimental ecology are also discussed.
\end{abstract}

\section{INTRODUCTION}

Il y a une quinzaine d'années, Wilson (1951) montrait l'existence d'une différence d'ordre biologique entre deux eaux de mer, l'une prélevée en Mer Celtique, l'autre aux environs de Plymouth. Il utilisait pour cette démonstration des larves de l'oursin Echinus esculentus dont les plutéus se développaient bien dans la première eau et mal dans la seconde. Au cours d'une sériẹ de travaux Wilson \& Armstrong (1952-1961) essayaient ensuite de retrouver et de préciser ce phénomène. Depuis 1957 nous avons également abordé ce problème des différences biologiques entre eaux de mer employant comme test biologique les plutéus de deux oursins communs en Méditerranée, Paracentrotus lividus et Arbacia lixula, et nous avons été amenés à étudier en détail le développement «in vitro» de ces plutéus (BougIs 1964a).

Nous avons ainsi obtenu une connaissance assez approfondie de la croissance des plutéus qui permet de les utiliser en laboratoire comme organismes-tests pour tenter d'élucider certains problèmes d'écologie expérimentale. Nous rejoignons ainsi une des préoccupations exposée il y a une dizaine d'années dans un autre symposium de biologie marine: mettre à la disposition des écologistes marins des organismes planctoniques pouvant être élevés «in vitro» et accessibles à l'expérimentation (RAE 1958).

\section{MÉTHODE}

La fécondation est toujours faite en eau de mer naturelle prise à l'entrée de la rade. Dans nos expériences les actions éventuelles des substances essayées s'exercent 
donc exclusivement sur le développement et la croissance et non sur les processus de la fécondation.

Les oeufs sont ensuite répartis dans des erlens placés en thermostat, dans 100 ou $200 \mathrm{cc}$ d'eau de mer à raison le plus souvent de 10 à 30 oeufs par cc. Pour suivre le développement des plutéus, des fractions de 5 à $10 \mathrm{cc}$ sont prélevéęs et filtrées sur filtres millipores de $25 \mathrm{~mm}$ de diamètre. Après lavage à l'eau distillée et séchage à $60^{\circ}$ les filtres sont montés dans l'huile de cédre qui, les éclaircissant, rend visible le squelette des plutéus; les mesures sont faites au microscope sur les baguettes composant ce squelette (Pressorr 1959). Nous mesurons la baguette somatique qui traduit le développement du corps même du plutéus et la baguette postorale qui sous-tend le bras postoral et permet d'apprécier le développement des bras. Pour simplifier, les mesures sont exprimées ici en divisions du micromètre (une division $=10,1 \mu$ ).

\section{ETAPES DE LA CROISSANCE}

Les larves d'oursins évoluent rapidement, en moins de $48 \mathrm{~h}$ à $25^{\circ}$, en plutéus à quatre bras (deux bras postoraux prolongeant les baguettes somatiques et deux bras antérolatéraux) élaborés grâce aux réserves de l'oeuf: c'est ce que nous avons appelé

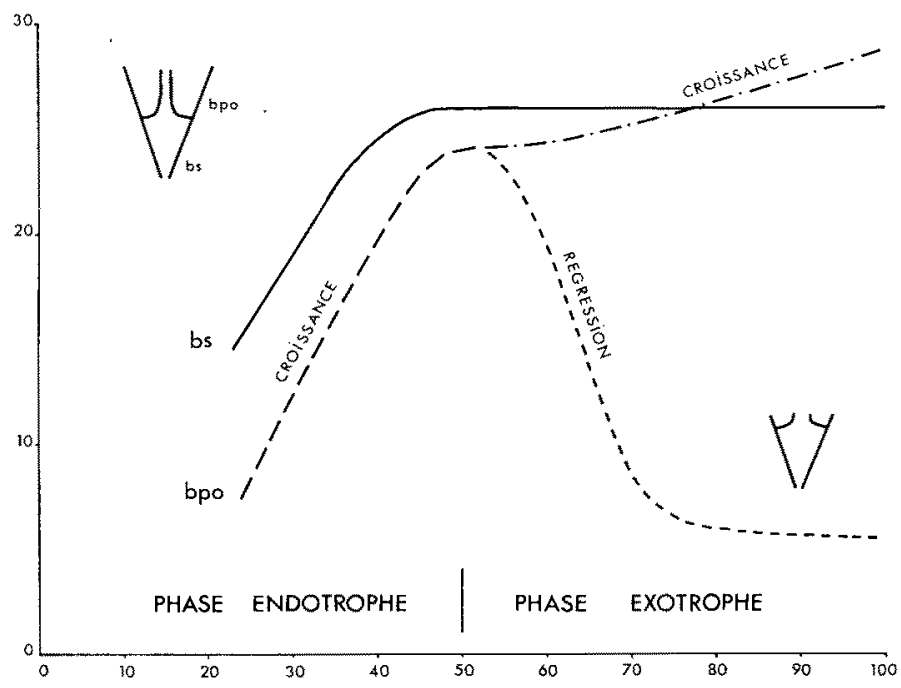

Fig. 1: Représentation schématique du développement des plutéus de Paracentrotus lividus. En abscisses temps en heures (à $\left.25^{\circ}\right)$ et en ordonnées division du micromètre $(1$ division $=$ $10,1 \mu) ; b s=$ baguette somatique; bpo $=$ baguette postorale

la $\mathrm{ph}$ a se end otrophe. Le plutéus doit ensuite, pour poursuivre son développement, trouver dans le milieu externe sa nourriture: c'est la phase exotrophe. Si la nourriture manque la larve soumise au jeûne ne meurt pas, mais ses bras vont diminuer, se résorber, jusqu'à disparaître presque complètement: c'est la régression. Sur la 
figure 1 nous avons représenté l'évolution de plutéus nourris et de plutéus soumis au jeûne en indiquant les phases précédentes. Ces courbes correspondent évidemment à des points moyens: les mesures réelles montrent une certaine dispersion que nous allons examiner de plus près.

\section{VARIABILITÉ ET VALIDITÉ DES MESURES}

Pour pouvoir utiliser comme test des différences dans la croissance des plutéus, plusieurs conditions sont nécessaires. (1) L'échantillon mesuré doit être assez réduit, pour des raisons pratiques, mais représentatif du lot étudié. (2) Des lots différents élevés dans les mêmes conditions doivent donner des résultats aussi concordants que possible; de l'écart entre ces lots dépendra en bonne part la précision des essais effectués par la méthode. (3) Pour une même série d'expériences une seule femelle et un seul mâle sont utilisés, mais d'une série à une autre, femelle et mâle diffèrent: il existe là une source de variation qu'il faut également apprécier.

Nous allons aborder ces différents points en distinguant successivement les baguettes somatiques et les baguettes postorales. Nos données concernent les plutéus de Paracentrotus lividus, espèce que nous avons utilisée le plus souvent.

\section{Baguettes somatiques}

La mesure des baguettes somatiques donne des courbes de fréquence montrant une distribution sensiblement normale; au bout de $45 \mathrm{~h}$ à $25^{\circ}$ le coefficient de variation $(C=s / \bar{x})$ se situe généralement de 0,05 à 0,08 et pour une longueur moyenne $(\bar{x})$ de 25, par exemple, la déviation standard (s) varie de 1,25 à 2 .

La détermination de l'intervalle de confiance de $95 \%$ en fonction de l'échantillon mesuré est donnée par le tableau suivant pour $\bar{x}=25, C=0,07, s=1,75$.

$\begin{array}{lccccc}\text { échantillon mesuré }(\mathrm{n}) & 30 & 60 & 90 & 120 & 150 \\ \text { erreur standard }\left(\mathrm{s}_{\overline{\mathrm{s}}}^{-}\right) & 0,32 & 0,23 & 0,18 & 0,16 & 0,14 \\ \text { intervalle }\left( \pm \mathrm{t}_{0,0,5} \cdot \mathrm{s}_{\overline{\mathrm{x}}}\right) & 0,65 & 0,46 & 0,36 & 0,32 & 0,28\end{array}$

Ainsi au delà de 90 mesures la précision n'est guère augmentée pour une dépense supplémentaire de temps assez grande: 30 mesures de la baguette somatique et de la baguette postorale nécessitent environ 15 minutes. Nous utilisons habituellement des échantillons de 60 ou 90 individus. Pour une première orientation, ou dans le cas de différences importantes, un échantillon de 30 individus peut même suffire.

En croissance exotrophe et en régression la variation des baguettes somatiques n'est pas sensiblement affectée.

La comparaison de lots différents élevés dans les mêmes conditions et provenant d'une même ponte fait apparaître quelques différences. Voici les longueurs moyennes des baguettes somatiques avec intervalles de confiance de $95 \%$ calculées pour 10 lots identiques (expérience $109,45 \mathrm{~h}$ à $25^{\circ}, \mathrm{n}=60$ ). 


$\begin{array}{lllllllllll}\text { moyennes }(\overline{\mathrm{x}}) & 26,03 & 26,18 & 26,12 & 26,43 & 26,37 & 25,85 & 25,90 & 25,37 & 26,23 & 26,65 \\ \overline{\mathrm{x}}+\mathrm{t}_{0,05} \cdot \mathrm{s}_{\overline{\mathrm{x}}} & 26,52 & 26,75 & 26,61 & 26,82 & 26,81 & 26,28 & 26,38 & 25,93 & 26,71 & 27,23 \\ \overrightarrow{\mathrm{x}}-\mathrm{t}_{0,05} \cdot \mathrm{s}_{\overline{\mathrm{x}}} & 25,54 & 25,61 & 25,63 & 26,04 & 25,93 & 25,42 & 25,42 & 24,81 & 25,75 & 26,07\end{array}$

L'analyse de variance donne les résultats suivants:

\begin{tabular}{|cccc|}
\hline Origine des variations & Somme des carrés & Degrés de liberté & Variance \\
\hline entre lots & 69 & 9 & 7,66 \\
résiduelle & 2154 & 590 & 3,68 \\
\hline Totale & 2223 & 599 & \\
\hline
\end{tabular}

On obtient donc $\mathrm{F}=7,66 / 3,68=2,09$.

Les tables donnant $F=2,45$, pour 9 et 590 degrés de liberté, avec un risque de $1 \%$, la différence n'est donc pas significative. Il faudra cependant veiller à s'entourer de toutes les précautions assurant la plus grande homogénéité entre les différents lots. De façon pratique il sera donc bon d'utiliser dans les expériences au moins deux lots identiques pour chaque traitement, si possible davantage, et d'effectuer une analyse de variance pour étudier les résultats. Ce n'est que dans les cas où les différences sont importantes que l'on pourra se satisfaire, entre un lot témoin et un lot traité, d'une simple comparaison des moyennes et des intervalles de confiance.

Enfin d'une femelle à une autre (fécondées par un même mâle) des différences notables existent, toutes autres conditions égales par ailleurs. Voici un exemple (expérience 112,45 h à $25^{\circ}, \mathrm{n}=60$ ):

$\begin{array}{lccccc}\text { Femelles } & \text { A } & \text { B } & \text { C } & \text { D } & \text { E } \\ \text { moyenne }(\bar{x}) & 25,68 & 25,22 & 26,45 & 27,30 & 26,70 \\ \overline{\mathrm{x}}+\mathrm{t}_{0,05} \cdot \mathrm{s}_{\overline{\mathrm{x}}} & 26,16 & 25,56 & 27,03 & 27,82 & 27,10 \\ \overline{\mathrm{x}}-\mathrm{t}_{0,05} \cdot \mathrm{s}_{\overline{\mathrm{x}}} & 25,20 & 24,88 & 25,87 & 26,78 & 26,30\end{array}$

L'analyse de variance donne les résultats suivants:

\begin{tabular}{|cccc|}
\hline Origine des variations & Somme des carrés & Degrés de liberté & Variance \\
\hline entre femelles & 164 & 4 & 41,00 \\
résiduelle & 973 & 295 & 3,29 \\
\hline Totale & 1137 & 299 & \\
\hline
\end{tabular}

On obtient $F=41,00 / 3,29=12,46$.

Avec un risque de $1 \% \mathrm{~F}$, pour 4 et 295 degrés de liberté, est égal à 3,38. La différence entre les femelles est donc significative.

Il est par conséquent nécessaire d'utiliser pour une série d'expériences une seule femelle (et un seul mâle) et d'introduire dans chaque série des lots de contrôle servant de témoins. 


\section{Baguettes postorales}

Comme pour les baguettes somatiques la mesure des baguettes postorales donne des courbes de fréquence sensiblement normales avec un coefficient de variation de 0,06 à 0,08 . Pour certaines femelles ce dernier peut être supérieur et atteindre 0,10 ce qui s'accompagne souvent d'une asymétrie de distribution du côté des valeurs inférieures à la moyenne. Dans les cas où cette asymétrie serait trop marquée il y aurait lieu de vérifier les résultats par de nouvelles expériences.

Avec la régression le coefficient de variation augmente considérablement atteignant 0,20 à 0,40 . En phase exotrophe il augmente aussi fréquemment: le tube digestif ne devient pas fonctionnel avant $40 \mathrm{~h}$ à $25^{\circ}$ et un temps de latence existe entre l'ingestion et l'utilisation, provoquant ainsi un début de régression avec accroissement de la variation.

Par ailleurs ce que nous avons indiqué pour les baguettes somatiques est valable dans ses grandes lignes pour les baguettes postorales.

\section{APPLICATIONS}

L'utilisation des plutéus comme organismes-test peut se faire soit en phase endotrophe soit en phase exotrophe: en phase endotrophe il s'agit surtout de modifications de croissance dues à des substances pénétrant dans la larve et freinant ou accélérant son développement; en phase exotrophe la longueur des bras répondant rapidement à la présence ou l'absence de nourriture il s'agira plus spécialement d'essayer des substances ou des souches nutritives. Nous allons examiner trois exemples d'utilisation.

\section{Effet toxique du cuivre à faible dose}

Nous avons montré (Bougis 1959-1965) que le cuivre additionné à l'eau de mer à des doses très faibles, de l'ordre de la dizaine de $\mu \mathrm{g}$ au litre, avait une action retardatrice sur le développement de la baguette somatique des plutéus. Cette action est bien illustrée par la figure 2. Le freinage de la croissance de la baguette somatique augmente de 0 à $20 \mu \mathrm{g}$ par litre de façon proportionnelle et en appelant I la longueur de la baguette somatique on a:

$$
1=1_{0}-\mathrm{kx}
$$

où $l_{0}$ est la valeur de 1 chez les témoins, $\mathrm{x}$ la concentration de cuivre en $\mu \mathrm{g} / \mathrm{l}$ et $\mathrm{k}$ un coefficient de l'ordre de 0,26 $( \pm 0,03)$. Cette relation permet d'envisager théoriquement un dosage biologique du cuivre. A la différence de la baguette somatique, la baguette postorale n'est pas affecté par le cuivre et sa croissance est même améliorée (figure 2), les bras bénéficiant des matériaux de réserve du plutéus laissés libres par le développement moindre des baguettes somatiques et du corps du plutéus. Avec $30 \mu \mathrm{g}$ au litre l'effet commence à porter sur les bras et $50 \mu \mathrm{g}$ par litre peut être considéré comme une dose létale. 


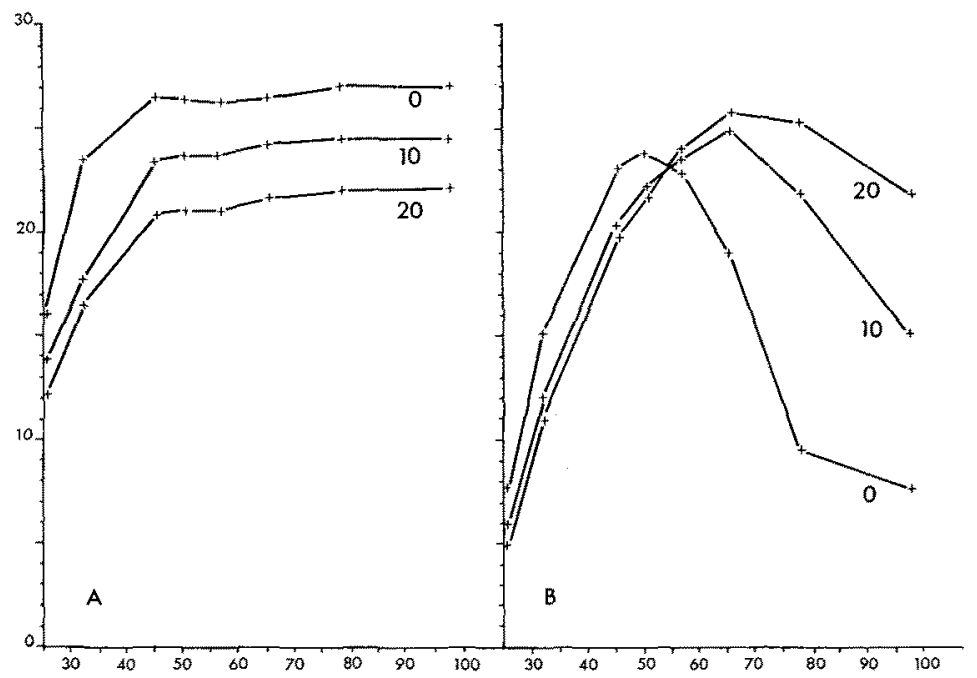

Fig. 2: Croissance des plutéus de Paracentrotus lividus élevés dans de l'eau de mer additionnée de 0,10 et $20 \mu \mathrm{g} / 1$ de cuivre (teneur initiale: $0,4 \mu \mathrm{g} / 1$ ); $\mathrm{A}$, baguette somatique; $\mathrm{B}$, baguette postorale. En abscisses temps en heures (à $25^{\circ}$ ) et en ordonnées divisions du micromètre (1 division $=10,1 \mu)$. Fécondation du 18 novembre 1964

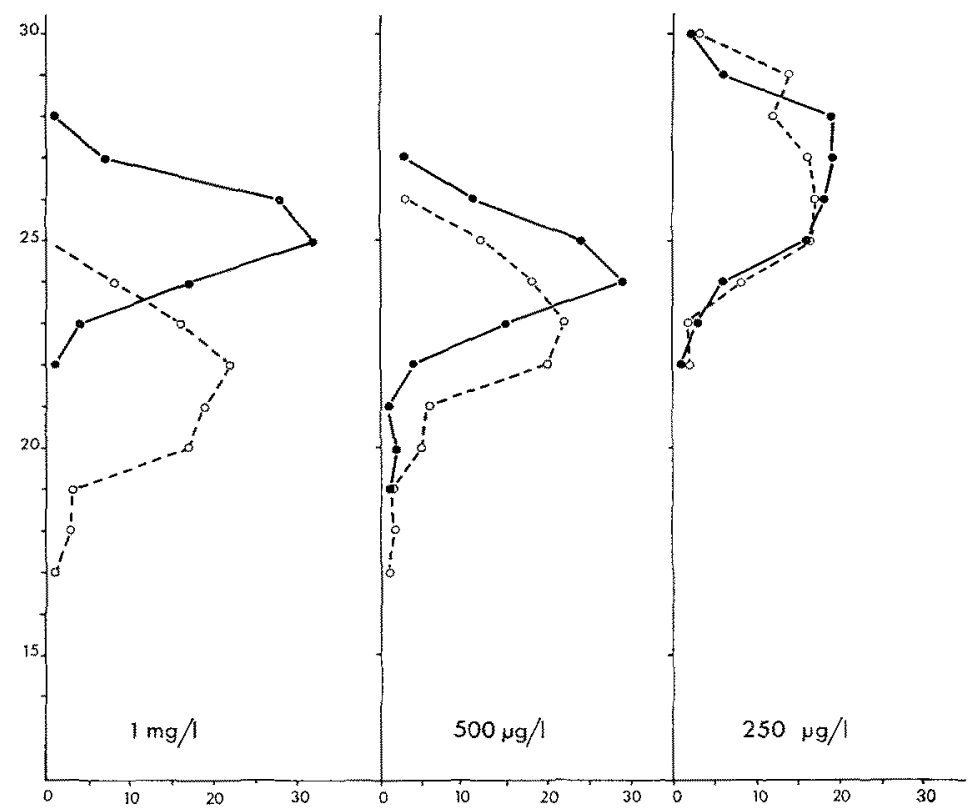

Fig. 3: Détermination du seuil toxique de la chloramine: en trait continu: lot témoin; en trait discontinu: lot expérimenté. En abscisses nombre d'individus et en ordonnées divisions du micromètre. Expériences du 26 octobre, 3 novembre et 24 novembre 1965 (3 femelles différentes). Température $=25^{\circ} \mathrm{C}$ 


\section{Détermination d'une solution bactéricide non toxique}

L'usage des bactéricides pour les élevages de larves marines se répand de plus en plus, la réduction du taux bactérien étant une des conditions du succès de ceux-ci. Dans les essais de nutrition d'organismes sur milieu synthétique les bactéricides sont également nécessaires.

Les doses à employer, aussi actives que possible vis à vis des bactéries, ne doivent évidemment pas perturber le développement des larves étudiées: il faut rester en dessous du seuil toxique. Les plutéus permettent de déterminer facilement de façon précise où se situe ce seuil. Ce ne sont cependant que des expériences comparables ultérieures qui feront connaître si ces seuils sont les mêtmes pour les larves d'autres groupes ou embranchements.

Voici à titre d'exemple les résultats obtenus pour la chloramine (Toluène sulfoné chloramide, sel de sodium) (figure 3). La dose de $1 \mathrm{mg} /$ l exerce une action toxique nette et freine notablement la croissance des baguettes somatiques, les baguettes postorales étant normales. La comparaison du lot expérimenté et du lot témoin donne $t=18,02$, c'est à dire que la différence est hautement significative $\left(t_{0,01}=2,6\right)$. Avec $500 \mu \mathrm{g} / \mathrm{l}$, $\mathrm{t}=5,66$, et pour $250 \mu \mathrm{g} / \mathrm{l} \mathrm{t}=0,23$. Le seuil d'action de la chloramine se situe donc entre 250 et $500 \mu \mathrm{g} / \mathrm{l}$.

\section{Détermination de la valeur nutritive d'une souche d'algue}

La seconde condition de succès des élevages de larves marines, en dehors de la réduction du taux bactérien, est la mise à leur disposition d'une nourriture satisfaisante. Pour les larves à alimentation végétale nous avons ainsi un nombre de plus en plus important de souches d'algues dont la valeur nutritive varie d'ailleurs dans des proportions assez grandes. Il serait intéressant, bien que cette valeur nutritive varie suivant les groupes, d'avoir à son sujet des informations objectives quantitatives. Les bras des plutéus qui répondent fidèlement, en phase exotrophe, aux conditions de nutrition permettent une telle détermination. Voici, à titre préliminaire, un exemple emprunté à une série d'expériences en cours.

La souche étudiée, Dunaliella marina, est ajoutée aux plutéus $45 \mathrm{~h}$ après la fécondation $\left(25^{\circ}\right)$. Les baguettes postorales sont mesurées au bout de 100 heures.

De la première série d'essais peut se déduire la relation suivante:

$$
1=1_{0}+0,015 x
$$

$1=$ longueur des baguettes postorales au bout de 100 heures; $1_{0}=$ longueur sans addition de Dunaliella, $\mathrm{x}=$ nombre de Dunaliella par plutéus, ajoutées à $45 \mathrm{~h}$. Dans ces conditions expérimentales une cellule de Dunaliella marina équivaut donc à une longueur de bras de plutéus de $0,15 \mu(1$ division du micromètre $=10,1 \mu)$. Il faut remarquer que l'effet ainsi mesuré correspond pour le plutéus à un effet similaire sur les deux bras postoraux et un effet du même ordre, sinon équivalent, sur les bras antérolatéraux. 


\section{PERSPECTIVES}

La disposition d'un organisme-test comme le plutéus d'oursin est susceptible évidemment de nombreuses autres applications en écologie expérimentale: nous en mentionnerons quelques-unes pour lesquelles nous avons des données préliminaires ou simplement des projets d'expériences.

Nous avons vu que les plutéus sont capables de réagir à l'addition à l'eau de mer d'une dizaine de microgrammes de cuivre au litre. En eau de mer pure, comme celle prélevée en rade de Villefranche, la teneur naturelle est de l'ordre du microgramme.

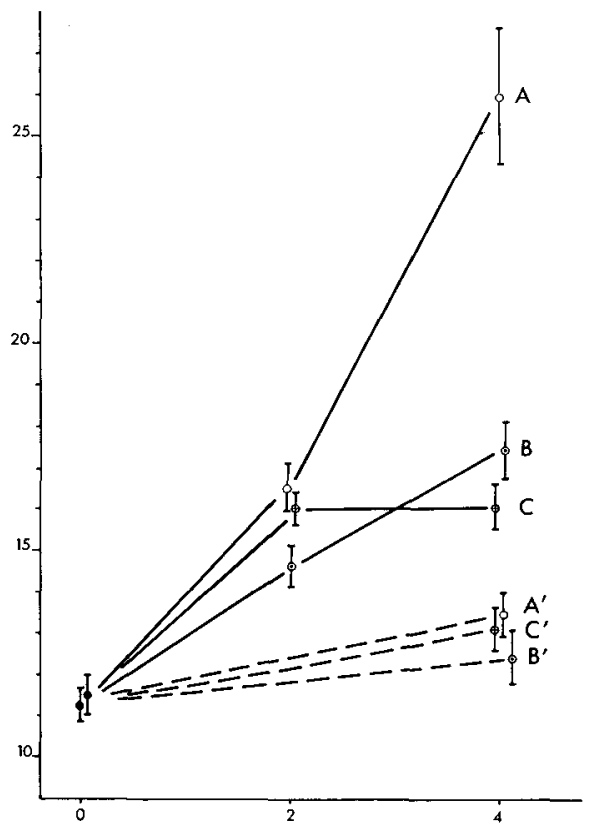

Fig. 4: Evolution de la longueur des baguettes postorales de plutéus régressés d'Arbacia lixula en fonction du temps (à $25^{\circ}$ ) dans différentes eaux: A, eau prise devant la Station au bord de la côte le 26-VIII-63 (Chlorophylle $\left.a=0,64 \mathrm{mg} / \mathrm{m}^{3}\right) ; B$, eau prise au fond de la rade le 26-VIII-63 (Chlorophylle $\left.\mathrm{a}=0,48 \mathrm{mg} / \mathrm{m}^{3}\right) ; \mathrm{C}$, eau prise à l'entrée de la rade le 26-VIII-63 (Chlorophylle $\mathrm{a}=0,13 \mathrm{mg} / \mathrm{m}^{3}$ ). $\mathrm{A}^{\prime}, \mathrm{B}^{\prime}, \mathrm{C}^{\prime}=$ mêmes eaux filtrées sur filtre millipore $\mathrm{H} A$ (mailles de $0,45 \mu$ ). En abscisses: temps en jours; en ordonnées divisions du micromètre (1 division $=10,1 \mu)$. Les intervalles de confiance $\left(\overline{\mathrm{x}} \pm \mathrm{t}_{0,05} \cdot \mathrm{s}_{\overline{\mathrm{x}}}\right)$ sont figurés

Dans le port même de Villefranche, par contre, la teneur atteint ou dépasse 10 microgrammes d'après les mesures faites au diéthyldithiocarbamate (S.D.D.C.). Dans une expérience préliminaire nous avons voulu vérifier si cette dose pouvait être retrouvée par le dosage biologique au plutéus. Or ce dernier essai n'a donné aucune différence par rapport aux témoins, ce qui semblerait indiquer que le cuivre présent est masqué pour les plutéus, peut-être par des substances complexantes: c'est là un ordre de recherches à poursuivre. 
Nous avons publié une note préliminaire (Bougrs 1964b) sur l'estimation de ce que nous avons appelé le phytoplancton efficace, c'est à dire la fraction du phytoplancton utilisable pour l'alimentation du zooplancton.

Nous utilisons comme test la régénération de plutéus régressés d'Arbacia lixula, plus commodes pour celà que ceux de Paracentrotus lividus. Nous avions ainsi trouvé en fin juin 1963 une différence considérable entre une eau tout à fait littorale, prise devant la Station, et une eau provenante de l'entrée de la rade, celle-ci étant plus pauvre. La figure 4 donne un second exemple de ce phénomène pour des eaux du 26 août dont la teneur en phytoplancton a été appréciée par P. Nival en mesurant la chlorophylle «a» retenue sur filtre de papier, à mailles de l' ordre de $10 \mu$. La régénération des bras observée n'est pas proportionnelle à cette teneur et, filtrées sur millipore $\mathrm{HA}$ (mailles de $0,45 \mu$ ), les trois eaux deviennent équivalentes et peu nutritives. Evidemment cette estimation du phytoplancton efficace n'est pas universelle, mais est liée strictement, dans l'état actuel de nos connaissances, au test utilisé.

Enfin pour de futures expériences de compétition entre différents organismes planctoniques concernant une nourriture donnée, les plutéus, avec ce que nous connaissons déjà de leurs exigences, représenteront un élément intéressant.

Mais bien d'autres utilisations peuvent encore être envisagées tirant parti d'une part de la connaissance améliorée de la croissance des plutéus et d'autre part de la possibilité, offerte par la fécondation artificielle des pontes d'oursins, de disposer de grandes quantités d'individus semblables.

\section{RESUME}

1. Utilisés pour l'étude des différences biologiques entre eaux de mer, les plutéus d'oursins (Paracentrotus lividus, Arbacia lixula) ont été étudiés en détail et constituent des organismes planctoniques pouvant être élevés «in vitro» et accessibles à l'expérimentation.

2. Après un bref rappel des méthodes les différentes phases du développement sont précisées: phase endotrophe, phase exotrophe et régression.

3. Les conditions d'utilisation expérimentale des plutéus sont étudiées. Des applications sont ensuite données: détermination de la toxicité de substances (cuivre, solution bactéricide) et évaluation de la valeur nutritive de cultures d'algues. Enfin sont évoquées quelques perspectives permises par les plutéus en écologie expérimentale.

\section{LITTÉRATURE CITÉE}

Bougis, P., 1959. Sur l'effet biologique du cuivre en eau de mer. C. r. bebd. Séanc. Acad. Sci, Paris 249, 326-328.

- 1961. Sur l'effet biologique du zinc en eau de mer. C. r. bebd. Séanc. Acad. Sci., Paris 253, 740-741.

- 1964a. Sur le développement des plutéus in vitro et l'interprétation du test de Wilson. C. $r$. bebd. Seanc. Acad. Sci., Paris 259, 1250-1253. 
- 1964b. Note préliminaire sur l'estimation du phytoplancton utilisable par le zooplancton (phytoplancton efficace) à l'aide d'un test biologique. Vie Milieu. (Suppl.) 17, 165-167.

- 1965. Effet du cuivre sur la croissance du plutéus d'Oursin (Paracentrotus lividus). C. $r$. bebd. Séanc. Acad. Sci., Paris 260, 2929-2931.

Pressorr, L., 1959. Contribution à la connaissance des Echinoplutéus de Paracentrotus lividus LMck, et Psammechinus microtuberculatus Blainv. Bull. Inst. océanogr. Monaco 56 (1142), $1-22$.

RaE, K. M., 1958. Parameters of the marine environment. In: Perspectives in marine biology. Ed. by A. A. Buzzati-Traverso. Univ. Calif. pr., Berkeley, 3-16.

Wilson, D. P., 1951. A biological difference between natural sea waters. J. mar. Biol. Ass. U.K. 30, 1-26.

- \& Armstrong, F. A. J., 1952. Further experiments on biological differences between natural sea waters. J. mar. biol. Ass. U. K. 31, 335-349.

- - 1954. Biological differences between sea waters: Experiments in 1953. J. mar. biol. Ass. U.K. 33, 347-360.

- - 1958. ... : Experiments in 1954 and 1955. J. mar. biol. Ass. U.K. 37, 331-348.

- 1961. ... : Experiments in 1960. J. mar. biol. Ass. U.K. 41, 663-681. 